\title{
Management of intracranial aneurysm in children: clipped and coiled
}

\author{
Mingqi Yang • Shuo Wang $\cdot$ Yuanli Zhao $\cdot$ Jizong Zhao
}

Received: 4 February 2008 / Published online: 24 May 2008

(C) The Author(s) 2008

\begin{abstract}
Objectives The objective was to describe the clinical features of intracranial aneurysm in children and evaluate the efficacy and safety of different treatment approaches for children with intracranial aneurysms.

Materials and methods Clinical data of 13 child patients with intracranial aneurysms that were diagnosed and treated in our hospital in the past 16 years were reviewed. Long-term follow-up data were also collected and reported.

Results Direct surgical treatment was performed on eight out of all 13 patients; among them, seven achieved good recovery and one died. The remaining five patients were treated with Guglielmi detachable coils (GDC). The aneurysm was successfully sealed off in one case and achieved good postoperative recovery. Residual aneurysm was detected in two cases; a second resection surgery was performed on one patient who died after the operation and on another with neck occlusion who recovered well. Two cases experienced aneurysm recanalization after interventional therapy; among them, one was treated with a second embolotherapy but had recanalization once again and was finally cured by aneurysm resection surgery.

Conclusion Aneurysmal surgery is proved to be a safe and effective treatment for children with intracranial aneurysm. Although requiring a long-term follow-up period to
\end{abstract}

M. Yang $\cdot$ S. Wang $\cdot$ Y. Zhao $\cdot$ J. Zhao $(\triangle)$

Department of Neurosurgery, Beijing Tiantan Hospital,

Capital Medical University,

No. 6 Tiantan Xili, Chongwen District,

Beijing 100050, China

e-mail: zhaojz@2008.sina.com determine the clinical outcome, GDC is also shown to be safe for a child having acute bleeding. Once a residual aneurysm or recanalization occurs, microsurgery is recommended.

Keywords Intracranial aneurysm .

Guglielmi detachable coils

\section{Introduction}

In child population, intracranial aneurysm has a very low incidence and the clinical manifestation is usually untypical. Additionally, the location and the size of intracranial aneurysm in children are often different from those of adult patients $[1-8,17]$. In this paper, we reviewed the clinical data of 13 child intracranial aneurysm patients that were treated in our hospital between January 1990 and December 2006.

\section{Materials and methods}

\section{Clinical data}

Patients A total of 13 child patients (nine males and four females) in the age range of 3-15 years old, five cases ranging from 3 to 6 years old and eight cases ranging from 6 to 15 years old, respectively, were included in the current study.

Clinical symptoms Six were acute cases with chief complaint of headache and/or dizziness. Their clinical manifes- 

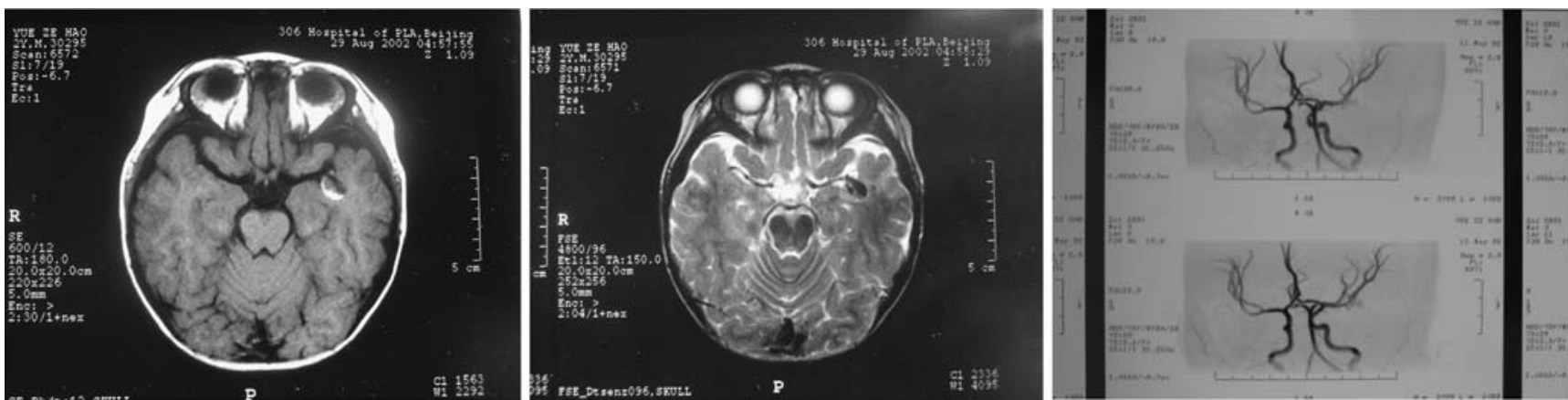

Fig. 1 Preoperative MRI and MRA (axial MR image revealing a medium-sized, similar round-shaped mass resembling aneurysm in the left temporal lobe); it was demonstrated as high density on T1- weighted image (left) and low density on T2-weighted image (center). MRA showing an abnormal bulking on the left middle cerebral artery (right) tations suggested intraventricular hemorrhage. Younger children could not make clear chief complaints but were dysphoric and irritable, whereas the most frequent chief complaint of older children was persistent and increasingly severe headache. Other symptoms were worst headache (four cases), vomiting (six cases), nausea (two cases), neck stiffness and neck pain (five cases), fatigue (one case), asthenia (two cases), muscular spasm of limbs (four cases), and low-grade fever (three cases). The remaining seven were chronic cases. Five of them had symptoms of monolateral asthenia, stagger, or holding immobilizing disability; one case had barylalia; one case had reduction in visual capacity with ptosis; and two cases experienced epileptic seizure. All 13 patients did not have an identified head injury or infection history. No family history of intracranial aneurysm or intracranial arteriovenous malformation (AVM) was reported.

Main physical signs Out of all 13 patients, six had neck rigidity and five had meningismus. One case reported monolateral oculomotor paralysis. Six patients had conscious disturbance of different degrees; among them, one was in a state of unconsciousness. Monolateral asthenia with Babinski's sign was found in seven patients. In the scale of Hunt and Hess, one patient was grade I, three were grade II, and two were grade IV.

Auxiliary examination

Lumbar puncture Bloody lumbar puncture was reported in five patients.

Imageology Cranial computed tomography (CT) scan was performed on all patients. Subarachnoid hemorrhage (SAH) was detected in five cases and intracranial hematoma was detected in one case. Magnetic resonance angiography (MRA) examination was performed on one case and an intracranial arterial deformation was detected (Fig. 1). Intracranial anticipate pathological changes were revealed in seven cases. Magnetic resonance imaging (MRI) examination was performed on six patients; among them, four patients were suggested to have intracranial aneurysms. Intracranial giant aneurysms were confirmed in four patients by $\mathrm{CT}$ angiography (CTA) examination.

Digital substract angiography (DSA) or 3D-DSA DSA was conducted in 13 patients within $1-3$ weeks after onset of the
Fig. 2 DSA and 3D-DSA obtained before first embolization (September 29, 2002). Left: DSA anteroposterior view showing a saccular aneurysm of the left middle cerebral artery. Right: 3D-DSA image of the left ICA demonstrating the presence of an aneurysm of the left middle cerebral artery

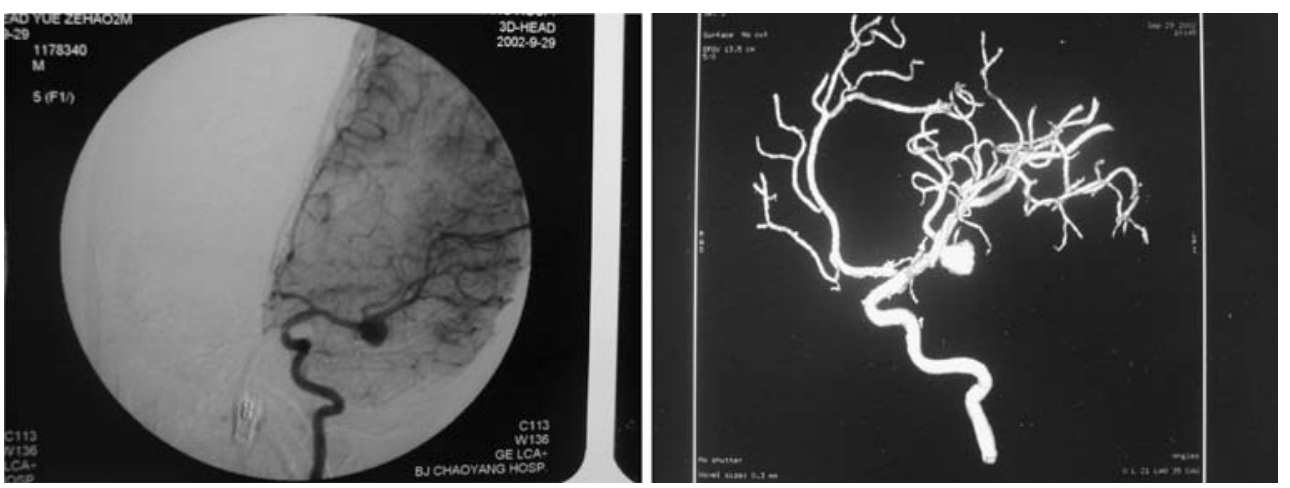




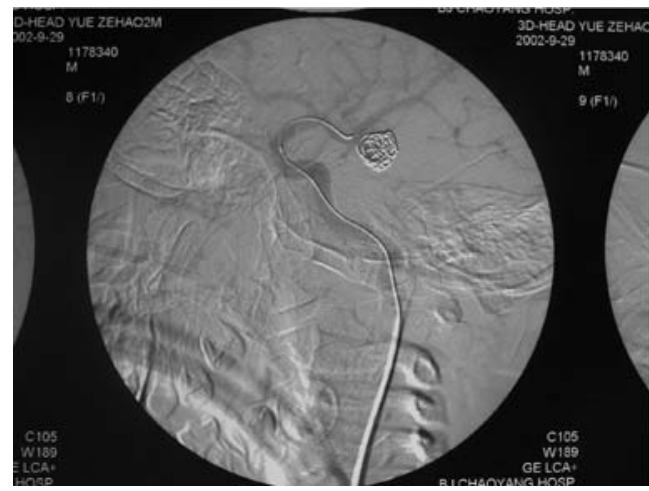

Fig. 3 DSA obtained after first embolization (September 29, 2002). Immediate post-procedure angiograms. Left: post-stent placement left carotid angiogram revealing complete, normal dilation of the stenotic portion of the ICA. However, the filling of the aneurysm has become

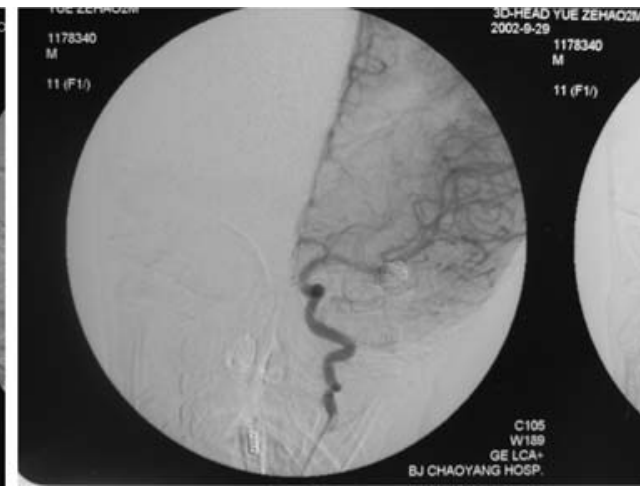

more obvious. Right: digital subtraction angiograms of left coronary artery obtained after stent placement and coil occlusion. There is complete obliteration of the aneurysm sacs disease. Among them, five cases were examined within 1 week and eight were examined within 2-3 weeks. All the patients were diagnosed as having an intracranial aneurysm. DSA or 3D-DSA also revealed abnormal bulking of arterial walls (Figs. 2, 3, 4, 5, 6, and 7). Thromb formation, adjacent artery angiospasm or stenosis and occlusion, and formation of compensatory circulation were observed in some of these patients. Complication of AVM was confirmed in one case by postoperative pathological examination.

\section{Treatment}

After the identification of diagnosis and obtainment of informed consent from the patients' family members, neurosurgeons and neurointerventional therapists cooperated in conducting the treatment programs. Surgical approaches included aneurysm neck occlusion, aneurysm neck ligation, occlusion of the carrying artery of aneurysm,

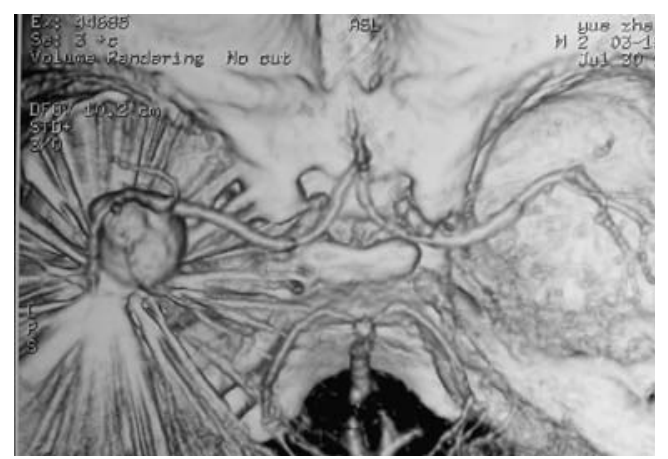

Fig. 4 Aneurysm recanalization and persisting overexpansion followup CTA obtained 10 months after first embolization (July 30, 2002) aneurysm resection surgery, aneurysm wrapping with muscle, and carotid artery ligation. Guglielmi detachable coil (GDC) embolization was conducted as the following procedure: After heparinization, a Fasguide guiding catheter was placed in the patient's femoral artery. An Excel 14microcatheter/Mizzen 10 microguide wire system (Boston Scientific/Target) was advanced through the guiding catheter and the tip of the microcatheter was positioned at the center of the aneurysm. All aneurysms were densely packed using GDC coils selected according to the size of the aneurysm. Angiograms were repeatedly performed during and after operation to obtain information about the positions of the coils and the degree of embolization (Figs. 8,9 , and 10).

\section{Results}

Follow-up visits have been conducted in all patients for more than 1 year. The Glasgow Outcome Scale standard was used for the evaluation of curative effect. Postoperative DSA was performed on three cases for aneurysm neck occlusion and three cases for aneurysm resection with aneurysm neck occlusion among the eight patients undergoing direct surgical treatment, which demonstrated a complete disappearance of the aneurysm. One patient treated with muscle wrapping and right internal carotid artery (ICA) ligation also recovered well and postoperative DSA revealed diminution in aneurysm size. Hematoma removal was performed on one case with aneurysm carrying artery occlusion and the patient died after the operation. Pathological examination demonstrated a complication of AVMs in this case. Among the five patients treated with GDC embolization, residual aneurysm was found in two cases; isolated aneurysm resection surgery 
Fig. 5 DSA and 3D-DSA

obtained before second embolization (August 7, 2003). Left:

DSA showing aneurysm recanalization and persisting overexpansion, oblique view. Right: 3D-DSA image of the left ICA demonstrating the presence of the aneurysm of the left middle cerebral artery and persisting overexpansion
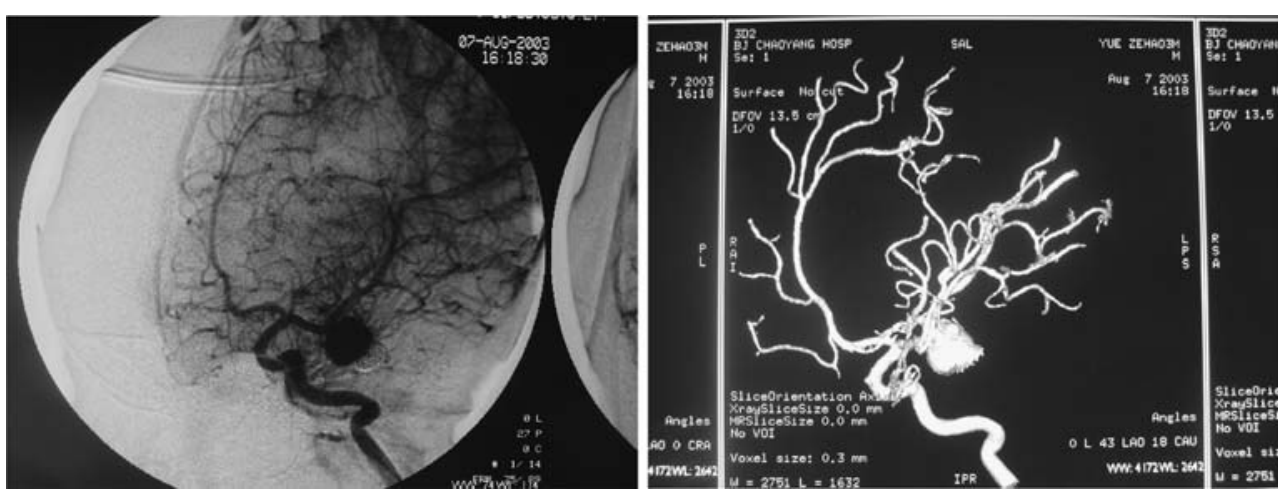

was performed on one patient who died after the operation and aneurysm resection surgery was performed on another with aneurysm neck occlusion and the patient recovered well. The aneurysms were successfully sealed off in three cases with a $100 \%$ degree of embolization. Follow-up visit at postoperative 1 year showed good recovery was achieved in one case but aneurysm recanalization occurred in the other two cases. A second embolization treatment was performed on one of the two cases but postoperative residual aneurysm was detected. Then the two cases received aneurysm resection surgeries and finally achieved satisfactory recovery. DSA examination demonstrated the complete disappearance of the aneurysm. All children with good recovery were able to undertake normal activities of daily living (Table 1).

\section{Discussion}

A child patient with intracranial aneurysm is relatively rare in clinical practice, usually having an incidence of $1-2 \%$ in all intracranial aneurysm cases $[1,4,5]$. The 13 child cases reported in the present study were about $1.4 \%$ of all intracranial aneurysm cases treated in our hospital during the same period. In 1849, Hare firstly reported an adolescent case with spontaneous SAH caused by a rupture of the posterior communicating aneurysm. Then in 1871, Eppinger reported a boy 15 years of age that fell ill during exercises and died 3 days later. Autopsy revealed a ruptured aneurysm of the anterior cerebral artery complicated with aortic stenosis. Therefore, although not so common in childhood and adolescence, SAH is a severe disease and should be managed carefully in clinical practice. In recent years, with the development of DSA technique and the clinical application of CTA and MRA techniques, the detection rate and also our understanding of aneurysm in children have been greatly improved.

In adult patients, aneurysm is the main cause of SAH, having a high death rate of $14 \%$ to $34 \%$ within $48 \mathrm{~h}$ after onset. In contrast to this, childhood aneurysm usually is symptomatic and often detected in DSA examination or even autopsy. Aneurysm has a higher incidence in boys than in girls and is mostly located at the furcation of ICA or vertebra-basilar artery posterior circulation. The incidence of large or giant aneurysms in child patients is higher than in adult patients whereas occurrence of aneurysm rupture is less, thus leading to a relative low death rate within $48 \mathrm{~h}$ after onset of the disease $[1,5,7,8]$. Out of the nine male cases and four female cases reviewed in this study, nine (69\%) had large or giant aneurysms. Among them, six
Fig. 6 DSA and 3D-DSA obtained after second embolization (August 7, 2003). Left: DSA anteroposterior view showing residual aneurysm of the left middle cerebral artery. Right: 3D-DSA image of the left ICA revealing the residual aneurysm of the left middle cerebral artery
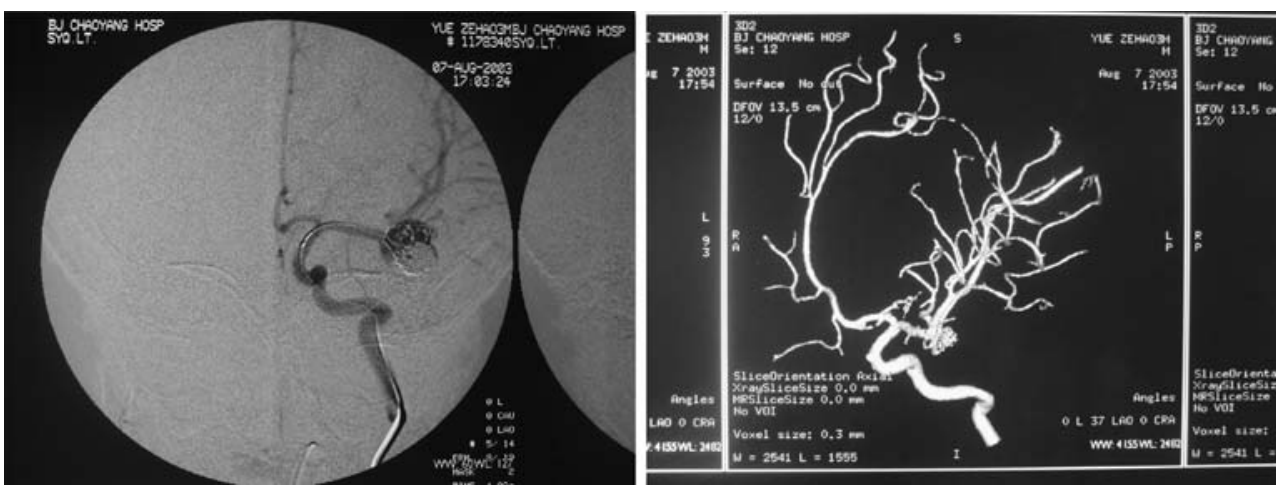
Fig. 7 DSA and 3D-DSA obtained 3 months after second embolization (November 21, 2003). Left: DSA anteroposterior view showing residual aneurysm and persisting overexpansion. Right: 3D-DSA image of the left ICA revealing the residual aneurysm
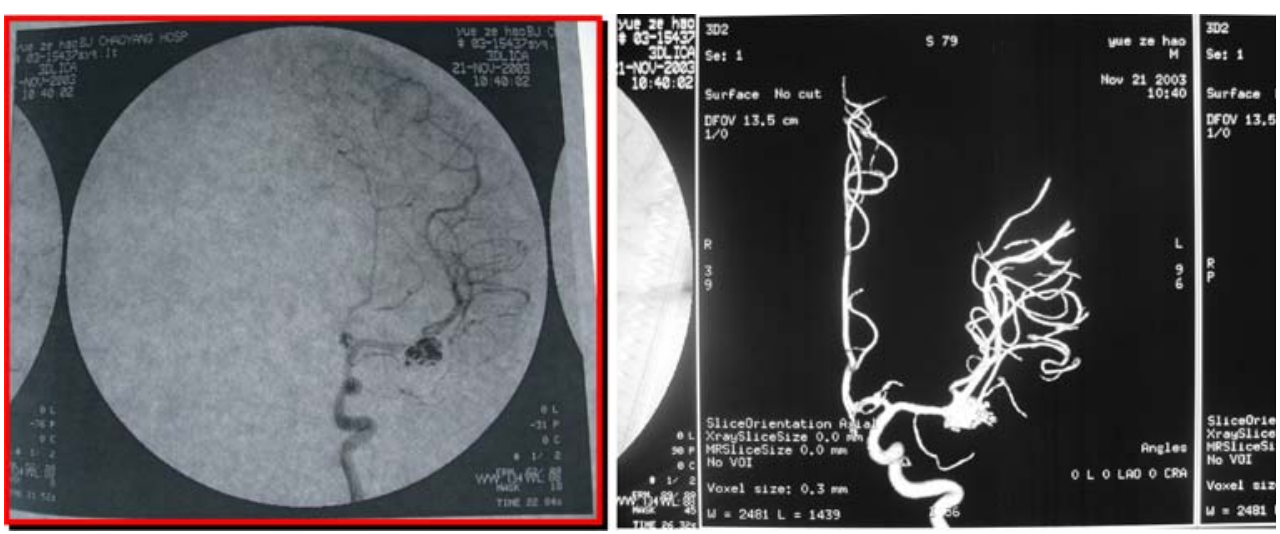

(46\%) patients initially suffered from hemorrhage and seven $(54 \%)$ had intracranial anticipate pathological changes. Evidence shows that multiple aneurysms seldom occur in child patients. The previous study indicated that the incidence of multiple aneurysms is in the range of $6-8 \%$ for child patients but was about $29 \%$ for adult patients. Taken together, these data suggest that there may be a difference in the pathological formation procedure of aneurysm between children and adults [1-6].

It has been confirmed that many factors including insufficiency or breakage of endovascular elastic layer and/or smooth muscle layer, either congenital structural defect or posterior degeneration (i.e., atherosclerosis, hypertension, or hemodynamic alterations), are implicated in the genesis of cystic intracranial aneurysm. The similarity of aneurysmal histology between children and adults indicates that the development of childhood aneurysm also involves these factors. Congenital factor is more prevalent than the others $[1,5,7-9]$ because other congenital diseases or vascular malformations in brain or peripheral tissues are often complicated in child patients. Stehbens [9] considered that the main cause of aneurysm is posterior degenerative changes; however, this is not the case in children because inflammatory changes or atherosclerosis is seldom observed in children and this may have been the explanation of the relative low incidence of childhood aneurysm. Some other studies also presumed that insufficiency of types III and IV collagen may be concerned with the development of childhood aneurysm [10]. Some viral or bacterial infections had also been confirmed in childhood aneurysms. All factors described above constitute the distinct characteristics of childhood aneurysm in size, location, and complications [1-11].

Due to the inadequate understanding of courses of childhood aneurysm rupture with profuse bleeding, the treatment selection was mainly based on knowledge coming from adult patients [7, 8]. Direct occlusion of the aneurysm neck is the optimal treatment prescription. It is noteworthy that childhood aneurysm of posterior circulation is often giant and fusiform in shape, making it lifethreatening to occlude the aneurysm directly. The best treatment approach would be gradually occluding the aneurysm carrying the vertebral artery. In Amacher and Drake's opinion [12], children in general have good tolerance to the operative procedure. Previous studies have demonstrated that children show good tolerance to the SAH with lower rates of mortality and deformity than adult
Fig. 8 Left: intraoperative photograph before clipping the aneurysm; a left MCA giant aneurysm is exposed. Right: intraoperative photograph after clipping the aneurysm; two aneurysm clips are applied and resected the aneurysm
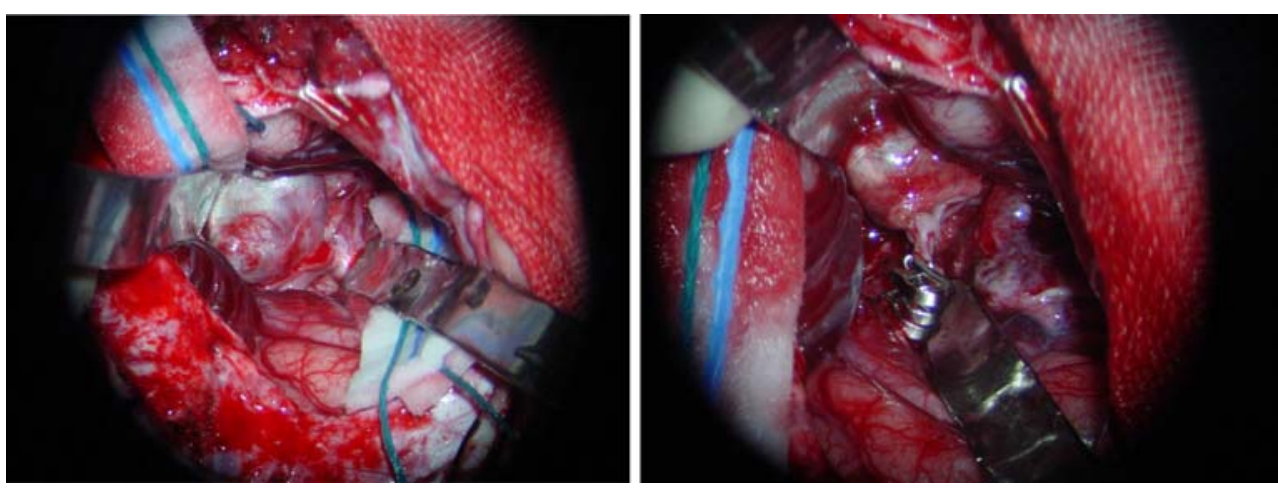


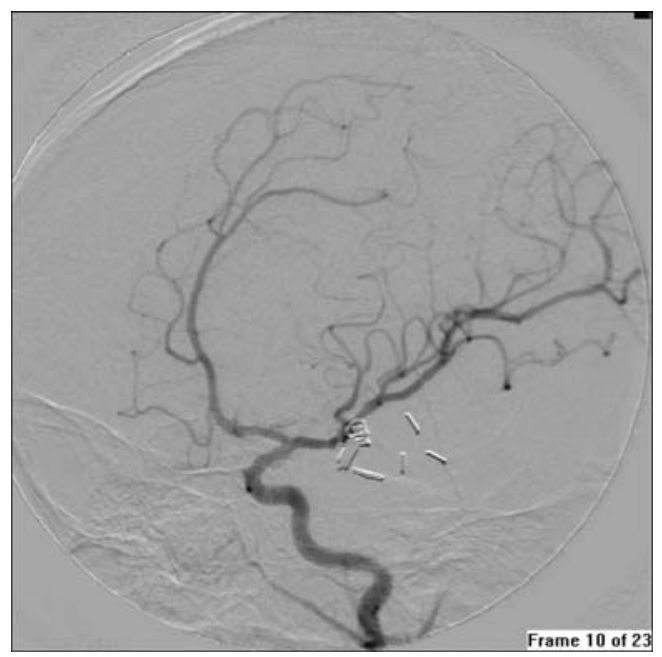

Fig. 9 Intraoperative angiogram obtained after clipping of the lesion. Oblique view of left ICA demonstrating that the aneurysm is obliterated, with preservation of the left MCA and its branches

patients. Cerebral infarction seldom occurs in child patients, even for those having severe cerebral angiospasm, whereas in adult patients the incidence of angiospasm-induced cerebral infarction is about $75 \%$. As reported by Amacher and Drake, the overall post-surgery mortality of child patients of aneurysm, including those with giant aneurysm, is less than $5 \%$.

GDC, which was designed and firstly used by Guglielmi (an Italian neurointerventional therapist), is a novel technique of aneurysm embolization. The invention and application of GDC greatly improved the treatment of intracranial aneurysm. It can occlude the aneurysm and ensure the unobstruction of the carrying artery simultaneously and finally achieve the outcome proximal to that of aneurysm occlusion surgery. According to Sluzewski's study [13], during the GDC embolization of intracranial aneurysm, the occurrence rate of aneurysm rupture and death rate were $2.5 \%$ and $1 \%$, respectively. Therefore, GDC embolization has been considered as a less invasive treatment and is becoming more acceptable than other approaches. Currently, GDC embolization has been commonly used and is a main approach in the treatment of adult patients with intracranial aneurysm. However, enough data about its safety and efficacy in child patients are still not available. Yu and his colleagues have reported successful application of GDC in the treatment of three children with intracranial aneurysms [14]. All three cases (two for posterior communicating aneurysms and one for anterior communicating aneurysms) had acute SAH caused by rupture of the aneurysm. The diameters of the aneurysm necks were in the range of $2.2-2.4 \mathrm{~mm}$ and the largest diameter of the aneurysm was less than $5.0 \mathrm{~mm}$. In the scale of Hunt and Hess, one patient was grade I, three were grade II, and two were grade IV. A $100 \%$ degree of embolization was achieved and clinical symptoms disappeared completely after the operation without reoccurrence of bleeding or any other complications. Follow-up visits have been carried out for all three patients for more than half a year (including two cases which had been followed up for more than 1 year). They demonstrated a good recovery and could undertake normal daily activity. DSA examination of two cases did not reveal residual aneurysm or recanalization. We consider that GDC embolization is safe for the treatment of child patients with intracranial aneurysms. It provides an alternative approach especially for those with acute rupture of aneurysm.

In our study, GDC embolization treatment was performed on a total of five child patients. The aneurysm was successfully sealed off in one male patient with acute aneurysm rupture (scale of Hunt and Hess, grade II). DSA
Fig. 10 Aneurysm specimen; its diameter is about $2.5 \mathrm{~cm}$
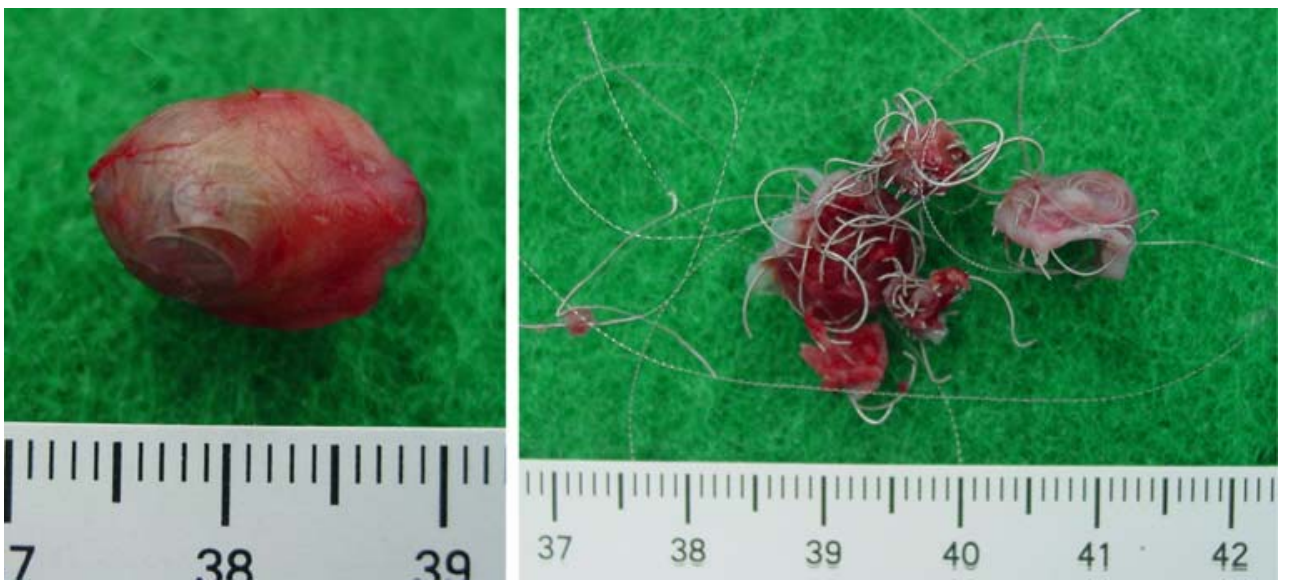
Table 1 Clinical data of 13 children with intracranial aneurysms

\begin{tabular}{|c|c|c|c|c|c|c|}
\hline \multirow{2}{*}{$\begin{array}{l}\text { Patient's } \\
\text { code }\end{array}$} & \multirow{2}{*}{$\begin{array}{l}\text { Aneurysm } \\
\text { position }\end{array}$} & \multirow{2}{*}{$\begin{array}{l}\text { Aneurysm } \\
\text { size }\end{array}$} & \multicolumn{2}{|l|}{ Clinical signs } & \multirow[t]{2}{*}{ Management } & \multirow[t]{2}{*}{ Outcome } \\
\hline & & & Hemorrhage & $\begin{array}{l}\text { Non- } \\
\text { hemorrhage }\end{array}$ & & \\
\hline 1 & LACoA & Small & SAH & & Aneurysm neck occlusion & Excellent \\
\hline 2 & LICAC2 & Small & SAH & & Aneurysm neck occlusion & Excellent \\
\hline 3 & LPCoA & Medium & $\mathrm{SAH}$ & & Aneurysm neck occlusion & Excellent \\
\hline 4 & LMCA & Large & & & $\begin{array}{l}\text { Aneurysm resection with aneurysm neck } \\
\text { occlusion }\end{array}$ & Good \\
\hline 5 & RMCA & Large & & & Aneurysm resection with aneurysm neck occlusion & Excellent \\
\hline 6 & RPICA & Large & SAH & & Aneurysm resection with aneurysm neck occlusion & Excellent \\
\hline 7 & RICAC2 & Large & & & Muscle wrapping, right ICA ligation & Good \\
\hline 8 & $\begin{array}{l}\text { LMCA and } \\
\text { AVMS }\end{array}$ & Giant & $\begin{array}{r}\text { Intracranial } \\
\text { hematoma }\end{array}$ & & $\begin{array}{l}\text { Hematoma removal, aneurysm carrying } \\
\text { artery occlusion }\end{array}$ & Dead \\
\hline 9 & RPCoA & Medium & $\mathrm{SAH}$ & & Interventional embolization & Excellent \\
\hline 10 & LPCA & Large & & & $\begin{array}{l}\text { Residual aneurysm after interventional } \\
\text { embolization, aneurysm resection with } \\
\text { aneurysm neck occlusion }\end{array}$ & Good \\
\hline 11 & LMCA & Giant & & & $\begin{array}{l}\text { Second interventional therapy, aneurysm } \\
\text { resection with aneurysm neck occlusion }\end{array}$ & Good \\
\hline 12 & RMCA & Large & & & $\begin{array}{l}\text { Recanalization after interventional therapy, aneurysm } \\
\text { resection with aneurysm neck occlusion }\end{array}$ & Good \\
\hline 13 & RVA & Giant & & & $\begin{array}{l}\text { Residual aneurysm after interventional embolization, } \\
\text { isolated aneurysm resection surgery }\end{array}$ & Dead \\
\hline
\end{tabular}

examination revealed a posterior communicating aneurysm on the right side; the aneurysm neck had a diameter of $2.4 \mathrm{~mm}$ and the aneurysm size was $5.0 \times 3.6 \mathrm{~mm}$. A $100 \%$ degree of embolization was obtained. DSA recheck did not find any residual aneurysm or recanalization. In one case with a giant vertebral aneurysm, post-embolizing residual aneurysm was detected. Isolated aneurysm resection surgery was then performed on the patient who died after the operation. Post-embolizing residual aneurysm was also detected in one case with a giant posterior cerebral aneurysm on the left side. For the remaining two cases, the first embolization surgery succeeded but followup visits revealed aneurysm recanalization. A second embolization treatment was performed on one of the two cases but postoperative residual aneurysm was reported again. Then, the two cases received aneurysm resection surgeries. DSA examination demonstrated that the aneurysm disappeared completely and good recovery was achieved.

Hayakawa [15] has reported the application of GDC in the treatment of 455 cases of intracranial aneurysms. The occurrence of postoperative residual aneurysm stem was $39 \%$ (178 cases). Of all patients with narrow stem small aneurysms, gradual thromb formation occurred in 12 cases $(50 \%)$, aneurysm recanalization occurred in eight cases (33\%), and no abnormality was found in the remaining eight cases. Of all patients with broad stem small aneurysms, gradual thromb formation and aneurysm recanalization were reported in six cases $(25 \%)$ and ten cases (42\%), respectively. In patients with large and giant aneurysms, the occurrence of recanalization was reported to be $87 \%$ (13 cases) and $90 \%$ (nine cases), respectively. Overall occurrence of gradual thromb formation and recanalization was respectively $25 \%$ (18 cases) and $49 \%$ (36 cases). Therefore, postoperative recanalization of residual aneurysm, especially for patients with broad stem large or giant aneurysms, was a very common event. As that child patient is more likely to have large or giant aneurysms, we consider GDC embolization to be more suitable and safe for children with small- or medium-sized aneurysms with acute rupture. However, to evaluate the long-term outcome of GDC treatment on children with large or giant aneurysms requires a longer follow-up period. Ausman [16] considered that occlusion of aneurysm by using a clip could help to draw close the aneurysm neck elastic layer which is the most burdening structure of the arterial wall, whereas embolization treatment could not do this at all. Therefore, the selection of treatment approach for child patients with large or giant aneurysms relies on the close cooperation of neurosurgeons and neurointerventional therapists. Follow-up DSA examination shall be enhanced for the cases having residual aneurysm necks. Once the residual aneurysm increases or recanalization occurs, microsurgical treatment is recommended. 
Open Access This article is distributed under the terms of the Creative Commons Attribution Noncommercial License which permits any noncommercial use, distribution, and reproduction in any medium, provided the original author(s) and source are credited.

\section{References}

1. Allison JW, Davis PC, Sato Y, James CA, Haque SS, Angtuaco EJC, Glasier CM (1998) Intracranial aneurysms in infants and children. Pediatr Radiol 28:223-229

2. Meyer FB, Sundt TM Jr, Fode NC, Morgan MK, Forbes GS, Mellnger JF (1989) Cerebral aneurysms in childhood and adolescence. J Neurosurg 70:420-425

3. Ferrante L, Fortuna A, Celli P, Santoro A, Fraiolo B (1988) Intracranial arterial aneurysms in early children. Surg Neurol 29:39-56

4. Humphreys RP (1989) Intracranial arterial aneurysms. In: Edwards MSB, Hoffman HJ (eds) Cerebral vascular disease in children and adolescents. Williams \& Wilkins, Baltimore, pp 247-254

5. Hacker RJ (1982) Intracranial aneurysms of childhood: a statistical analysis of 500 cases from the world literature. Neurosurgery 10:775 (Abstract)

6. Herman JM, Rekate HL, Spetzler RF (1991) Pediatric intracranial aneurysms: simple and complex cases. Pediatr Neurosurg 17:66-73

7. Proust F, Toussaint P, Garnieri J, Hannequin D, Legars D, Houtteville JP, Freger P (2001) Pediatric cerebral aneurysms. J Neurosurg 94:733-739
8. Pasqualim A, Mazaa C, Cavazzani P, Scineza R, Dapian R (1986) Intracranial aneurysms and subarachnoid hemorrhage in children and adolescents. Child Nerv Syst 2:185-190

9. Stehbens WE (1982) Intracranial berry aneurysms in infancy. Surg Neurol 18:58-60

10. Pope FM, Nicholls AC, Narcisi P (1989) Some patients with cerebral aneurysms are deficient in type III collagen. Lancet 1:973-976

11. Husson RN, Saini R, Lewis LL (1992) Cerebral artery aneurysms in children infected with human immunodeficiency virus. J Pediatr 121:927-930

12. Amacher AL, Drake CG, Ferguson GG (1981) Posterior circulation aneurysms in young people. Neurosurgery 8:315-320

13. Sluzewski M, Bosch JA, van Rooij WJ, Nljssen PCG, Wijnalda D (2001) Rupture of intracranial aneurysms during treatment with Guglielmi detachable coils: incidence, outcome, and risk factors. J Neurosurg 94:238-240

14. Yu JM, Fan GP, Zhong WX (2002) Interventional treatment of intracranial aneurysm in children. J Chin Clin Med Imaging 13:161-163

15. Hayakawa M, Murayama Y, Duckwiler GR, Gobin YP, Guglielmi G, Vinuela F (2000) Natural history of the neck remnant of a cerebral aneurysm treated with the Guglielmi detachable coils system. J Neurosurg 93:561-568

16. Ausman JI (1998) Cerebral aneurysm: clip or coils. Surg Neurol 50:395-402

17. Zhao J, Sun H, Wang S (2006) Neuroendoscope-assisted minimally invasive microsurgery for clipping intracranial aneurysms. Min Inv Neurosurg 49:335-341 